\title{
INFLUÊNCIA DA VELOCIDADE E DIREÇÃO DO VENTO NA ESPÉCIE COCOS NUCIFERA VAR. TYPICA (ARECACEAE) EM ÁREAS COSTEIRAS NATURAIS E URBANAS
}

\author{
Ingrid Juliane Machado de Melo ${ }^{1}$, Otacilio Antunes Santana ${ }^{1}$, Euzelina dos Santos Borges Inácio ${ }^{1}$, Laerte \\ Bezerra de Amorim², José Imaña Encinas ${ }^{3} 4$
}

\section{RESUMO}

A influência do vento sobre indivíduos de Cocos nucifera var. typica em duas áreas de estudo, natural e urbana, foi o objetivo principal desta pesquisa. Nas áreas de estudo foram analisados vinte coqueiros, segundo os critérios meteorológicos, como velocidade do vento em $\mathrm{m} / \mathrm{s}$ e biométricos como volume do tronco, número de folhas e ângulo de inclinação. Anemômetros em ambas as áreas foram dispostos para auferir a velocidade do vento nos sentidos sertão-litoral e litoral-sertão. As áreas de estudo foram determinadas a partir da presença ou ausência de barreiras, num raio mínimo de $200 \mathrm{~m}$, que impedissem a livre circulação do vento em todos os sentidos. A área natural escolhida foi a Praia de Maria Farinha, no litoral Norte e a Praia de Boa Viagem, litoral Sul de Pernambuco. Esta última foi selecionada como área urbana devido a presença de prédios e construções próximos à costa. A partir deste estudo, foi observado a grande influência exercida pelo vento no desenvolvimento e crescimento dos indivíduos de Cocos nucifera var. typica encontrados na área urbana. A presença de barreiras e impedimentos à passagem do vento, no sentido sertão-litoral, promove uma resultante vetorial no sentido litoral-sertão o que gera forte impacto sobre a estrutura da planta.

Palavras-chave: coqueiro; volume do tronco; ângulo de inclinação.

\section{INFLUENCE OF WIND SPEED AND DIRECTION IN COCOS NUCIFERA VAR. TYPICA (ARECACEAE) IN NATURAL AND URBAN COASTAL AREAS}

\begin{abstract}
The influence of wind on Cocos nucifera var. typica in two different areas, natural and urban, was main aimed of this research. Twenty coco trees in both areas were analyzed according to meteorological variable, in this case wind speed in $\mathrm{m} / \mathrm{s}$, and biometric variables such as trunk volume, number of leafs and inclination angle. Anemometers in both areas were placed to measure wind speed in two directions: continent-coast and coastcontinent. The areas of study were determined by the presence or the absence of barriers, in a radius of 200 $\mathrm{m}$, that block the wind flow in both directions. Maria Farinha Beach, North coast of Pernambuco, was chosen as natural area and Boa Viagem Beach, South coast of Pernambuco was chosen as urban area, due to the presence of buildings near the coast. From this study, it was possible to observe the influence of wind on development and growth of individuals of Cocos nucifera var. typica found in urban area. The presence of buildings near those trees block the continent-coast wind which promotes a resulting vector in coast-continent way, causing several impacts to tree structure.
\end{abstract}

Key words: coco tree; wind; trunk volume; inclination angle.

\footnotetext{
${ }^{1}$ Departamento de Biofísica e Radiobiologia, Centro de Ciências Biológicas, Universidade Federal de Pernambuco. ingrid.melo27@gmail.com; otacilio.santana, euzelina.inacio[ufpe.br].

${ }^{2}$ Departamento de Energia Nuclear, Centro de Tecnologia e Geociências, Universidade Federal de Pernambuco.

laerteamorim@yahoo.com.br

${ }^{3}$ Departamento de Engenharia Florestal, Faculdade de Tecnologia, Universidade de Brasília. imana@unb.br

${ }^{4}$ recebido em 26.05.2012 e aceito para publicação em 05.12.2013
} 
O coqueiro (Cocos nucifera L.) é uma espécie tropical amplamente cultivada no mundo, encontrada em todas as regiões intertropicais. É uma espécie de uso múltiplo por meio de várias partes da planta, como a raiz, o estipe, a inflorescência, as folhas, o palmito e, principalmente, o fruto, razão pela qual também tem sido denominada de árvore da vida (WADT, 1997). Siqueira (2002) descreveu que no Brasil a cultura do coqueiro está disseminada numa área de 247 mil hectares com uma produção aproximada de 1,1 bilhão de frutos, concentrada no Nordeste do país. A maior parte $(83 \%)$ da produção brasileira destina-se à indústria alimentícia, e nos Estados do Espírito Santo e do Rio de Janeiro basicamente destinada para o consumo da água de coco (WADT, 1997).

$\mathrm{O}$ coqueiro gigante foi introduzido pela primeira vez no Brasil em 1553, no Estado da Bahia e procedente das ilhas de Cabo Verde. A origem remota deste material seria a Índia ou Sri Lanka de onde cocos teriam sido introduzidos em Moçambique. Esta hipótese é confirmada pela semelhança entre o coqueiro do Oeste da África e o coqueiro Gigante de Moçambique (SIQUEIRA, 2002).

O Cocos nucifera possui número somático de cromossomos igual a 32 e número haplóide igual a 16, sendo, portanto, uma espécie diplóide. Grupos de coqueiro são distinguidos em dois: coqueiro gigante (var. typica) e coqueiro anão (var. nana), sendo denominados, na literatura, de variedades (WADT, 1997). Cocos nucifera var. typica é, predominantemente de polinização cruzada devido às flores masculinas se formarem antes das flores femininas. As plantas têm estipe, com circunferência média de $84 \mathrm{~cm}$ e atingem, em média, altura de 18 metros. Elas apresentam crescimento rápido, atingem a maturidade aos 6-7 anos de idade, produzem 50 a 80 frutos grandes por planta ao ano, e permanecem produtivas por mais de 70 anos. As folhas têm comprimento médio de 5,5 metros. O florescimento é tardio e ocorre normalmente entre seis e oito anos após o plantio (SIQUEIRA, 2002).

Nos coqueiros e nas plantas em geral, embora essas sejam organismos aparentemente estáticos, estão presentes notáveis capacidades de adaptação perante o seu meio envolvente e reage aos diferentes estímulos exteriores através de vários movimentos denominados de tropismos e nastismos. Os tropismos são movimentos progressivos permanentes, geralmente lentos e orientados num determinado sentido por ação de estímulos exteriores, enquanto os nastismos são movimentos que não são orientados em relação à fonte de estímulo, e sim dependente da simetria de seus tecidos vegetais (ZEIGER; LINCOLN, 2006).

A luz é um dos fatores ambientais que mais influi no desenvolvimento vegetal, seja através de fotoestimulação da biossíntese de substâncias, fototropismo, fotomorfogênese ou fotoperiodismo (CASTRO, 2005). Iino (2001) definiu fototropismo como a curvatura de movimentos, cujo sentido é determinado pela direção ou distribuição desigual da luz incidente. Existem dois tipos de fototropismo, o positivo e o negativo. Segundo este autor, o positivo é quando o movimento ocorre em direção à luz (por exemplo, caule), e o negativo em caso contrário (por exemplo, raiz).

Além da luz, um fator de extrema importância no crescimento das plantas é o vento, que tem sido considerado como importante fator ecológico em florestas, devido ao seu papel no transporte de vapor de água, na energia térmica, nos grãos de 
pólen, nos esporos e sementes de plantas,e na geração de eletricidade estática por seus efeitos na evaporação e transpiração (ZHU et al., 2004).

$\mathrm{O}$ vento move-se nas direções horizontal e vertical, e afetado pelas condições das superfícies que ele encontra. Geralmente, a extensão superficial do vento atinge 50 aos $100 \mathrm{~m}$ acima da superfície da Terra e é marcado por fortes misturas de massas de ar, como por exemplo, as turbulências. O vento ocasiona efeitos significantes na fotossíntese. Quando o fluxo de ar passa através das árvores e move as folhas, o ar é bombeado para dentro e para fora dos estômatos, o que acelera a troca de $\mathrm{CO}_{2} \mathrm{e}$ $\mathrm{O}_{2}$ (ZHU et al., 2004).

É importante na determinação fenotípica das árvores, pois afeta a morfologia das raízes, caule, copa e também a distribuição do crescimento (ZHU et al., 2004). Peltola (2006) constatou que sob um regime de vento, em condições constantes de velocidade e direção, a estabilidade mecânica da árvore é afetada pela força horizontal desempenhada pelo vento e pela força vertical gravitacional, causando uma inclinação em mecânica para adaptação ao ambiente, e melhor posicionamento para suas atividades metabólicas (captação de luz, controle hídrico, etc.) . Alguns estudos têm mostrado o impacto ambiental e econômico causado pela ação de ventos fortes na arborização urbanas, como, redução das taxas fotossintéticas, trocas gasosas e bioacumulação de carbono, redução na produção de frutos e área foliar (sombra), e queda de árvores e tecidos vegetais nas vias (SILVA et al., 2007; DUNTEMANN, 2006; STIGTER et al., 2002; CORDERO, 1998).

A análise quantitativa do crescimento é o primeiro item na avaliação da produção vegetal. Para tanto, a quantidade de material contido na planta, como caule, raízes e frutos, e a superfície fotossintetizantes (área foliar), devem ser mensurados (MONTEIRO et al., 2005). De acordo com Goyal et al. (2013), a área foliar representa o aparato de interceptação de luz para a fotossíntese e a partir da dessa estimativa é possível encontrar algumas variáveis ecofisiológicas como razão de área foliar, taxa assimilatória líquida, taxa de crescimento e desenvolvimento e quantificação de variações no crescimento das plantas, devido a diferenças genéticas ou ambientais.

Neste contexto, foi hipotetizado para esta pesquisa que: a velocidade e direção do vento influenciam mais na biometria da espécie Cocos nucifera var. typica em áreas costeiras urbanas do que em áreas costeiras naturais. Então os objetivos desta pesquisa foram: i) quantificar a velocidade e direção do vento em duas áreas costeiras: uma natural e outra urbana e ii) analisar a influência do vento na biometria (número de folhas, volume e inclinação do tronco) dos indivíduos de Cocos nucifera var. typica em áreas costeiras urbanas e naturais.

\section{MATERIAIS E MÉTODOS}

A área de estudo e amostragem dos dados foram áreas costeiras identificadas como naturais e urbanas (Figura 1). Classificou-se de naturais aquelas que ocorriam livres da passagem do vento em todos os sentidos em um raio mínimo de $200 \mathrm{~m}$ (Figura 1A) e as áreas urbanas aquelas em que o vento foi bloqueado dentro deste raio por construções (edifícios e casas; Figura 1B).

Figura 1. Localização das áreas de amostragem dos dados: A) Praia de Maria Farinha e B) Praia de Boa Viagem. Ingrid Juliane Machado de Melo et al. 


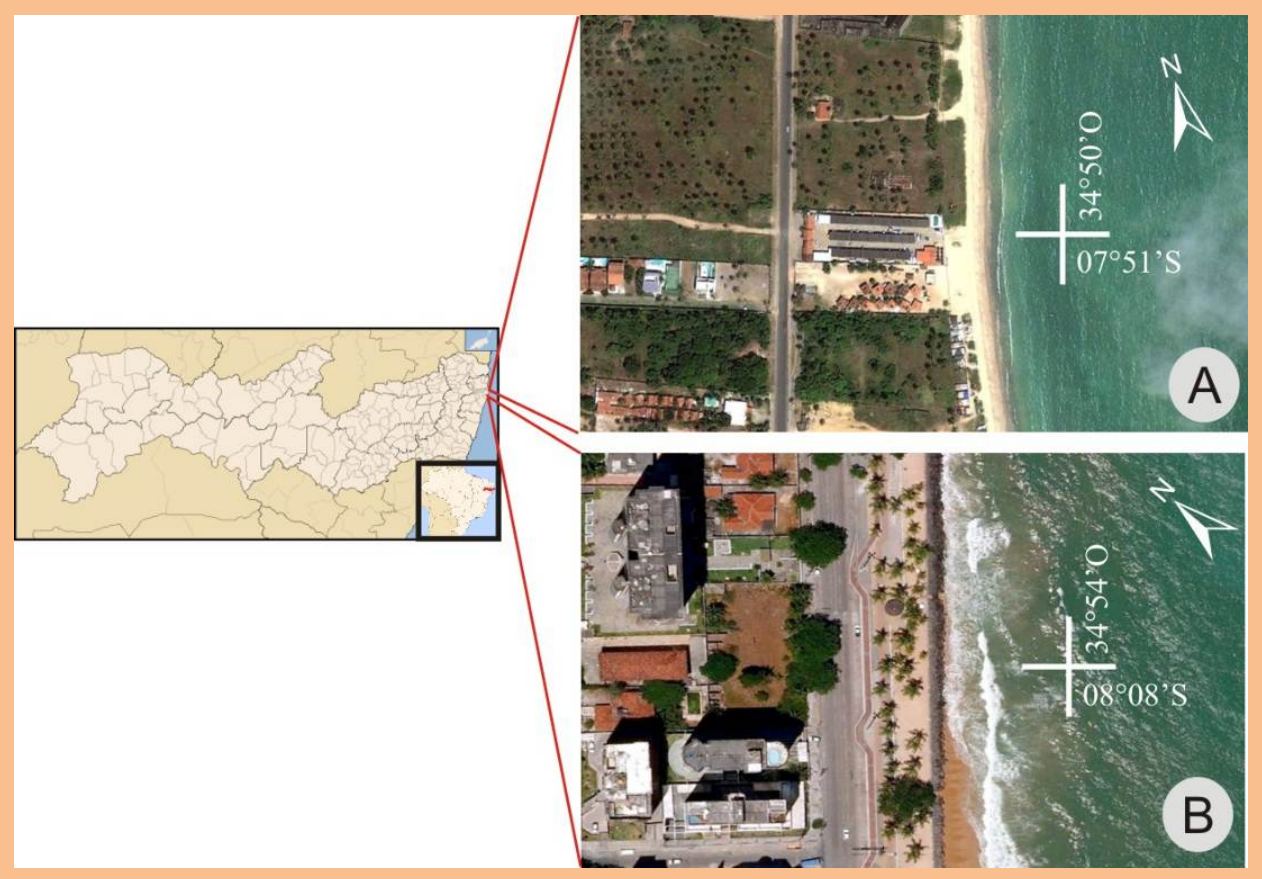

Os quatro anemômetros (WS40 Series Wind Speed Sensor - Environdata) (Figura 2), pertencentes à base meteorológica do Instituto Nacional de Meteorologia (INMET), dispostos no local a $500 \mathrm{~m}$ de distância um do outro, foram utilizados nas áreas de estudo, para mensuração das respectivas variáveis: velocidades $(\mathrm{m} / \mathrm{s})$ e direção do vento, sendo instalados dois na praia e dois no sentido sertão, conforme representado na Figura 3, a uma altura de 3 metros. Os anemômetros foram calibrados segundo manual do fabricante. Os dados de cada minuto das 24 horas dos dias de janeiro a outubro de 2011 que foram armazenados em um datalogger (Eko21N - Ekopower) e recuperados no site do INMET e analisados.

Figura 2. A) Base meteorológica do Instituto Nacional de Meteorologia (INMET) e B) Anemômetro.
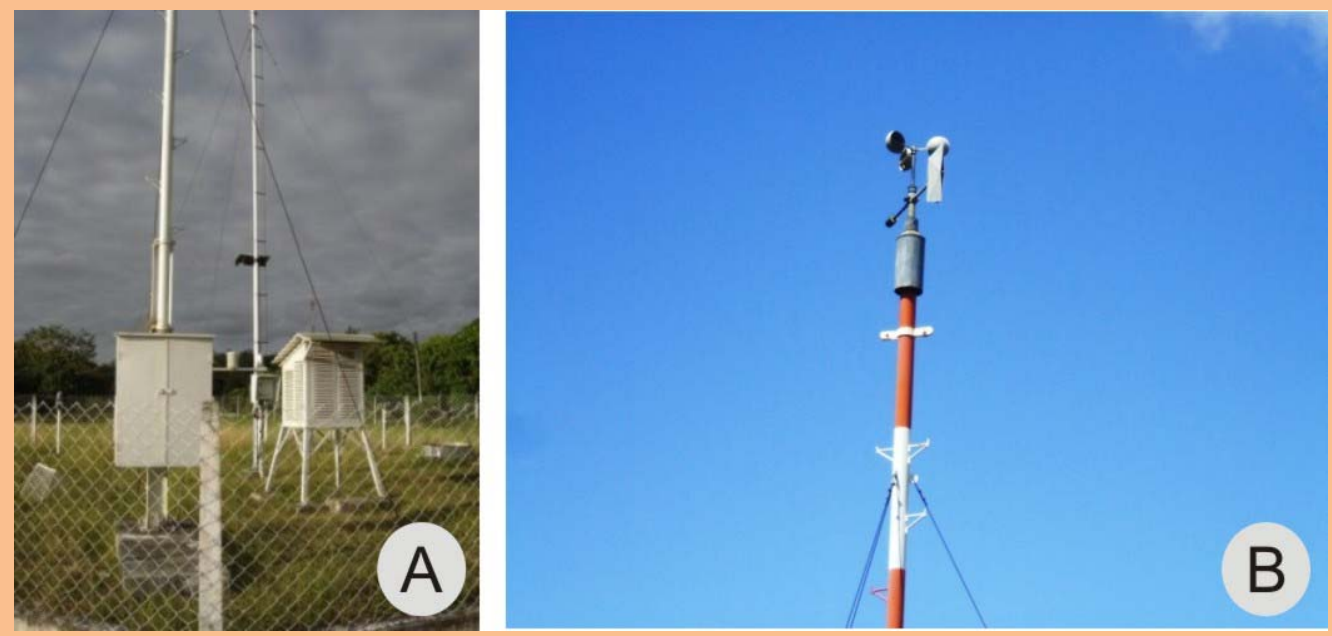
Para determinação do valor da média da velocidade do vento anual nas áreas foi utilizado a seguinte equação (VAN STAN II et al.,2011):

$\Delta \mathrm{V}=\mathrm{Vap}-\mathrm{Vas}$

Eq. 1

em que, $\Delta \mathrm{V}=$ variação da velocidade do vento $(\mathrm{m} / \mathrm{s})$; Vap = velocidade média anual do vento amostrado no anemômetro da praia e Vas = velocidade média anual do vento amostrado no anemômetro mais próximo do sertão.

A espécie Cocos nucifera var. typica (Aracaceae) foi avaliada quanto à biometria: volume do tronco e número de folhas. $\mathrm{O}$ volume do tronco de 100 indivíduos de C. nucifera var. typica; sendo 50 em áreas costeiras naturais e 50 em urbanas, foi calculado a partir da equação:

$\mathrm{Vol}=\pi \cdot\left(\frac{d}{2}\right)^{2} \cdot h \cdot f f$

Eq. 2

em que, $\mathrm{Vol}=$ volume do tronco visível na parte aérea da planta, $\mathrm{d}=$ diâmetro da circunferência do tronco dos indivíduos arbóreos, mensurados à altura do peito $(1,30 \mathrm{~m}), \mathrm{h}=$ altura da árvore e $\mathrm{ff}=$ fator de forma, correspondente a relação entre o volume real do tronco de um indivíduo vegetal pelo volume do cilindro da mesma altura e diâmetro da área basal, (Figura 4). O ff (fator de forma) para a espécie em questão é de 0,53 (RAHMAN; KHAN, 2007).

Figura 3. Posição dos anemômetros na praia. AS = anemômetro sertão e AP anemômetro praia.

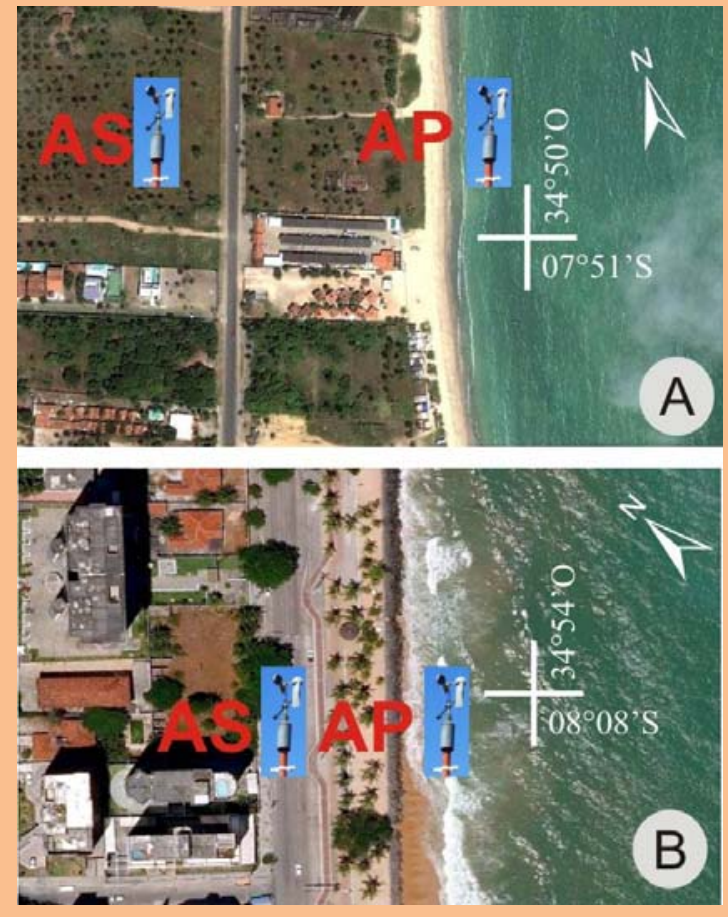

Ingrid Juliane Machado de Melo et al. 
Figura 4. Representação do cálculo do fator de forma: volume real do tronco dividido pelo volume do cilindro.

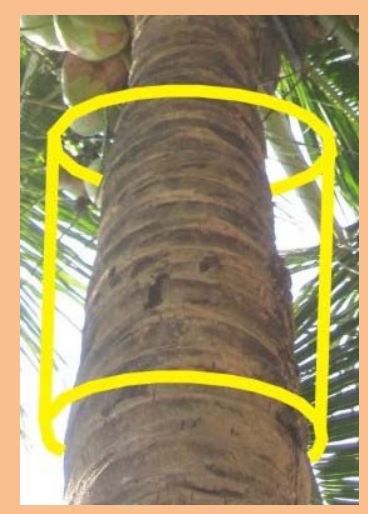

Os indivíduos também foram avaliados segundo sua inclinação e altura. A obtenção do ângulo de inclinação, conforme demonstrado na Figura 5, foi realizada através de fotografias de cada indivíduo que foram avaliadas no programa CorelDraw X5 com uso de um transferidor, enquanto que a variável altura foi obtida através do método de referência de altura, conforme a Figura 6.

Figura 5. A) Amostragem da resultante para o cálculo do ângulo de inclinação do indivíduo de Cocos nucifera var. typica (Aracaceae) e B) Registro através do programa CorelDraw X5.
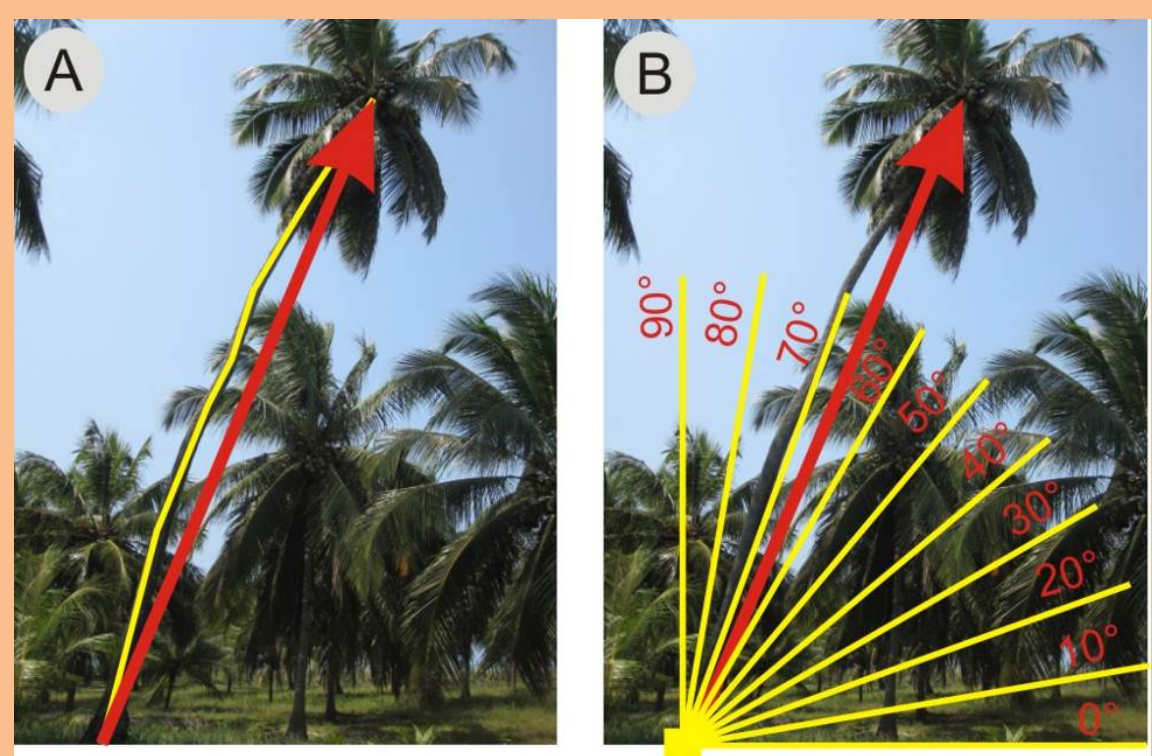
Figura 6. Método de referência de altura.

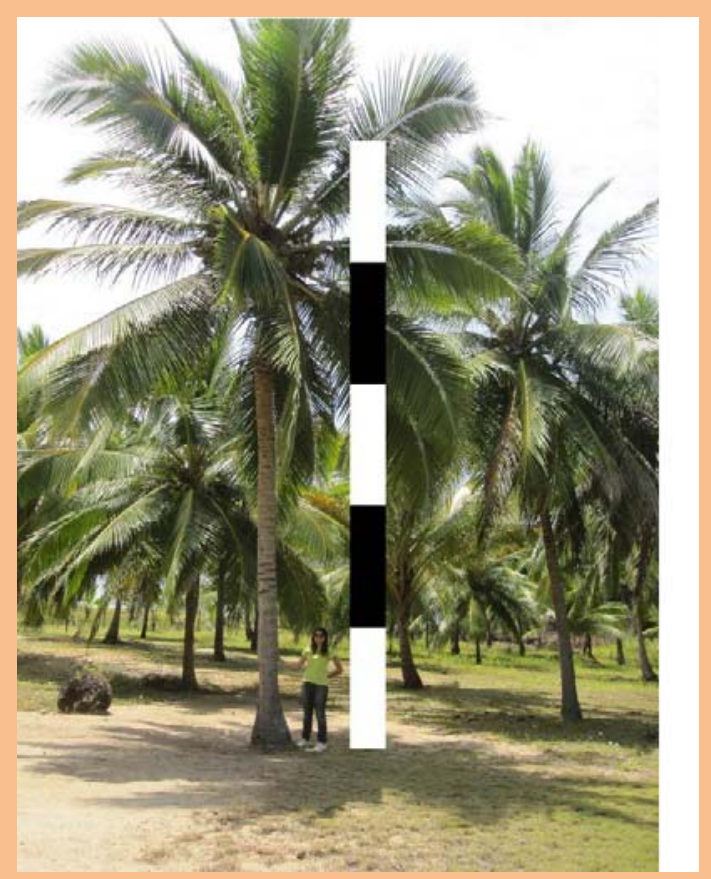

Outra variável mensurada para verificação da vegetais amostrados foi o número de folhas vivos influência do vento na morfologia dos indivíduos produzidos (Figura 7) por cada indivíduo registrado.

Figura 7. Seta representa a identificação para contagem dos folhas dos indivíduos de Cocos nucifera var. typica (Aracaceae).

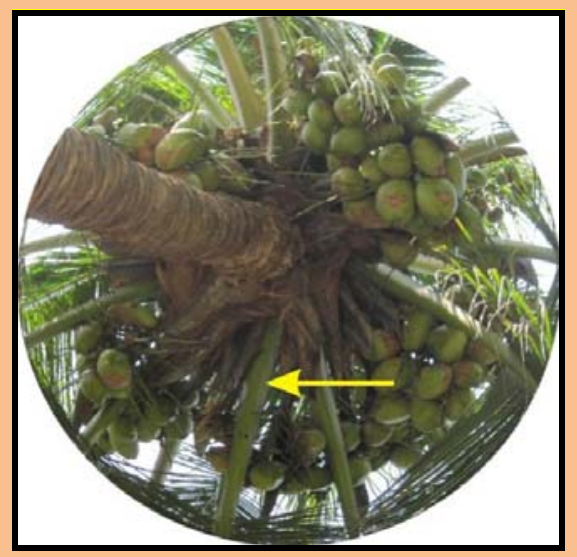

Teste de hipótese não paramétrica Mann-Whitney (ZAR, 2010) foi efetuado para obtenção do nível de significância (p) e com isso verificar a diferença significativa entre os grupos amostrais: i) área costeira natural e ii) área costeira urbana, em todas as variáveis analisadas. A análise de correlação de Spearman $(\rho)$ foi efetuada para se observar a relação entre as variáveis meteorológicas e biológicas avaliadas. 
A velocidade média resultante do vento foi maior na área urbana do que na área natural e essa diferença significativa (Tabela 1). Isto se deve pelo fato de que na área natural não houve impedimento antrópico para o vento em nenhuma direção como na área urbana, através das construções. Estas barreiras fizeram com que a direção do vento resultante da área urbana modificasse, em relação à área natural (Tabela 1), o que confirma a influência que os prédios exercem na variável meteorológica avaliada (Figura 8).

No tocante às variáveis biométricas avaliadas (Tabela 1), todas elas apresentaram diferenças significantes em relação às áreas estudadas. Todas apresentaram resultados médios inferiores na área urbana. Outro fator biométrico de destaque foi à inclinação do tronco dos indivíduos de Cocos, o qual apresentou ângulos mais acentuados nas áreas urbanas (Figura 8).
As plantas possuem um crescimento em direção à luz e como a luz natural do sol, em seu movimento diário, apresenta uma resultante de incidência perpendicular em relação ao solo, as plantas tenderiam a crescer de forma perpendicular (TAKAHASHI; BADGER, 2011). A eliminação de outros fatores como a precipitação, que também tende a atingir o solo perpendicularmente e a temperatura, o vento seria o único fator determinante na inclinação e na redução dos valores das variáveis mensuradas (VAN STAN II et al., 2011), conforme observados na Tabela 1. Quanto às variáveis volume do tronco e número de folhas, constatam-se valores inferiores nos indivíduos presentes na área urbana. Esta redução dos valores do volume de tronco e do número de folhas (Figura 9) aponta a resposta ecofisiológica das espécies vegetais frente às variáveis ambientais (ARTECA; ARTECA, 2011).

Tabela 1. Resultado das variáveis meteorológicas do vento e biométricas dos indivíduos de Cocos nucifera var. typica (Aracaceae), em áreas naturais: Praia de Maria Farinha e áreas urbanas: Praia de Boa Viagem. Valor de p resultado do teste não paramétrico Mann-Whitney.

\begin{tabular}{lccc}
\hline \multicolumn{1}{c}{ Variável } & Naturais & Urbanas & p \\
\hline$\Delta \mathrm{V}(\mathrm{m} / \mathrm{s})$ & $8,70 \pm 3,10$ & $12,5 \pm 5,20$ & $<0,001$ \\
$\Delta$ direção (Vento) & NO (Noroeste) & O-NO (Oeste-noroeste) & \\
Tronco $\left(\mathrm{m}^{3}\right)$ & $0,30 \pm 0,08$ & $0,22 \pm 0,12$ & 0,003 \\
Folhas $(\mathrm{n})$ & $18,0 \pm 2,94$ & $12,1 \pm 4,21$ & $<0,001$ \\
$\theta\left(^{\circ}\right)$ & $77 \pm 3,0$ & $62 \pm 12$ & $<0,001$ \\
$\Delta$ direção (Cocos) & NO & NO & \\
\hline
\end{tabular}

Esta pesquisa corrobora com outros que confirmaram a influência de áreas com altos valores de velocidade do vento e mudança de direção na redução da bioacumulação em plantas (ARTECA;
ARTECA, 2011; BUNCE, 2011) e na redução de processos fisiológicos vegetais como fotossíntese e respiração (MIRANDA et al., 2011; DUARTE et al., 2005; STIGTER et al., 2002). 
Outro fator observado na Praia de Boa Viagem, correspondente à área urbana, foi a influência antrópica direta, ou seja, o contato direto com o coqueiro, que promoveu afilamentos do tronco e pode ter contribuído para redução das variáveis biométricas nessa área. Na Figura 10 é possível observar, por exemplo, um afilamento no indivíduo de Cocos, causado pela fixação de uma faixa publicitária.

A influência do vento nas variáveis biométricas em áreas urbanas foi confirmada também pelo resultado da análise de correlação entre as variáveis meteorológicas e biométricas. Nas Tabelas 2 e 3, o resultado de " $r$ " demonstrou que as relações foram significativas $(r>0,5)$ em todas as análises, porém na área urbana (Tabela 3) o valor de " $r$ " foi maior do que na natural, o que demonstra a dependência da variável biológica mensurada para a meteorológica.

Os resultados de $\mathrm{r}<0$ revelam a relação inversamente proporcional entre as variáveis meteorológicas e biológicas, por exemplo, quanto maior a velocidade média do vento menor o volume de tronco dos indivíduos avaliados (Tabelas 2 e 3 ). Estas relações foram também citadas pela literatura (ARTECA; ARTECA， 2011; BUNCE， 2011; MIRANDA et al., 2011; DUARTE et al. 2005; STIGTER et al., 2002).

Figura 8. Direção e Velocidade do vento (A e B) e direção e inclinação (C e D) dos indivíduos de Cocos nucifera var. typica (Aracaceae), em áreas naturais: Praia de Maria Farinha (A e C) e áreas urbanas: Praia de Boa Viagem (B e D).

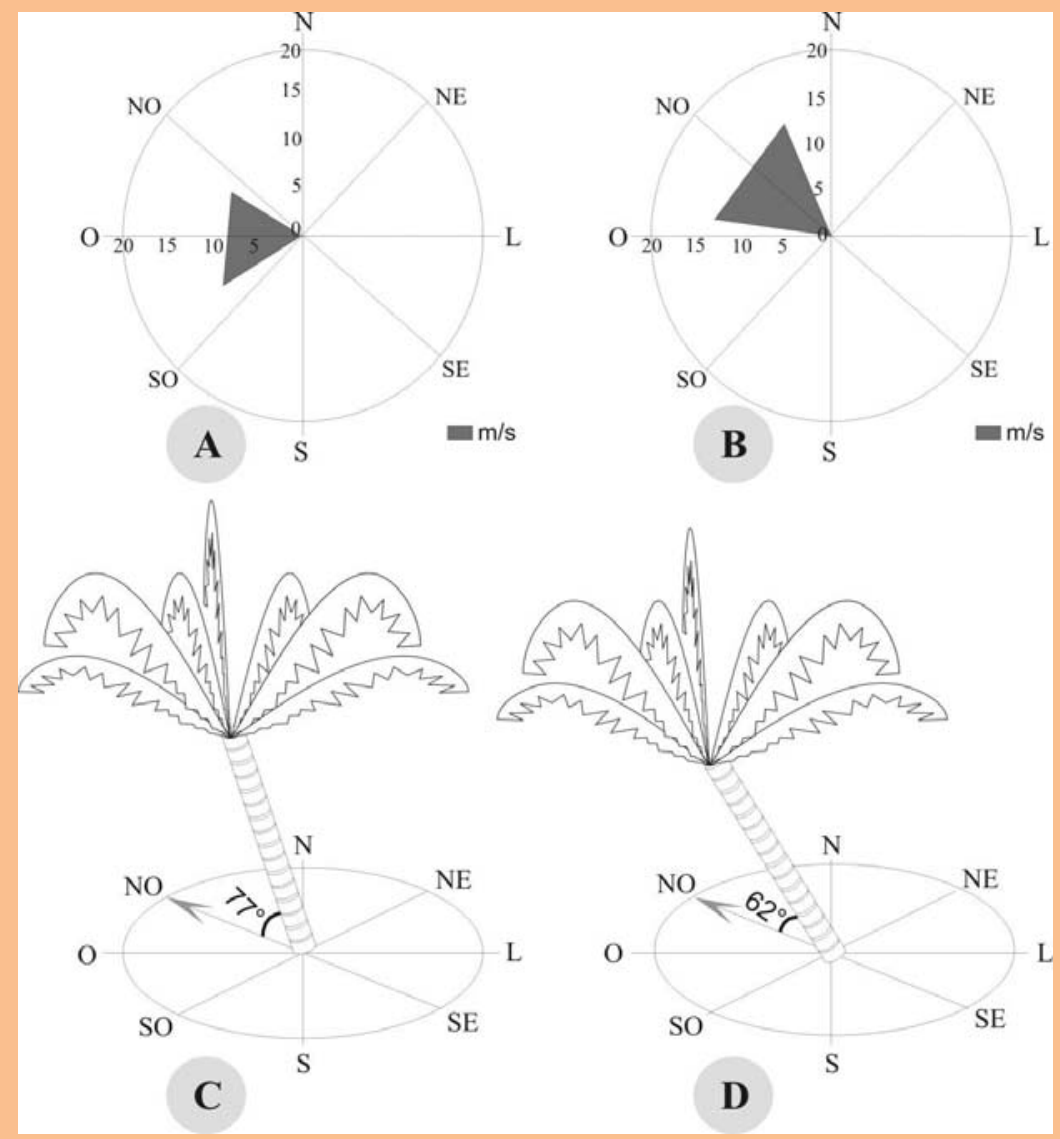

Ingrid Juliane Machado de Melo et al. 
Figura 9. Visualização das copas (A e B) e dos troncos dos indivíduos de Cocos nucifera var. typica (Aracaceae), em áreas naturais: Praia de Maria Farinha (A e C) e áreas urbanas: Praia de Boa Viagem (B e D).
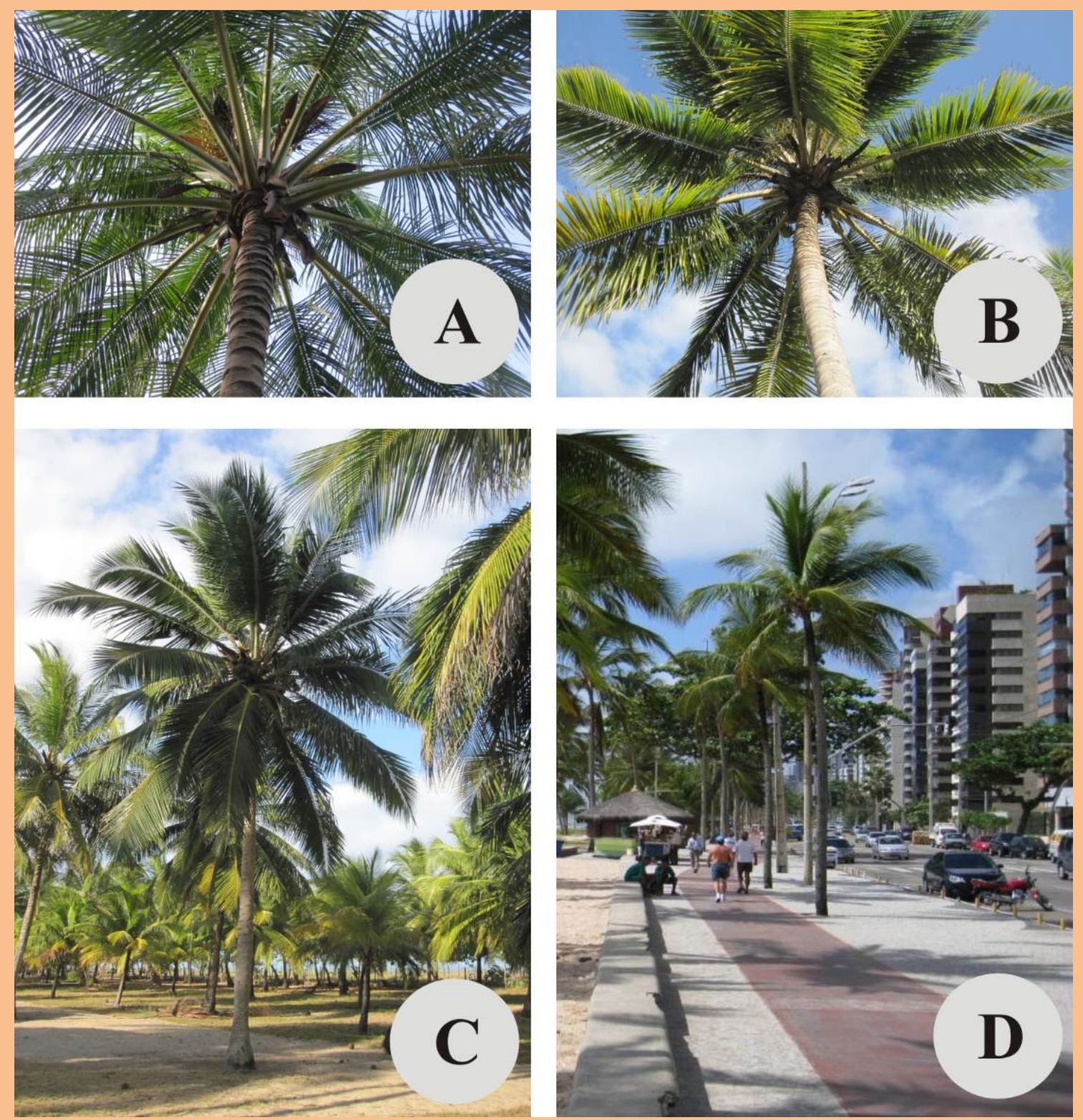

INFLUÊNCIA DA VELOCIDADE E DIREÇÃO DO VENTO NA ESPÉCIE COCOS... 
Figura 10. Visualização de afilamento de um indivíduo de Cocos nucifera var. typica (Aracaceae) causado por ação antrópica na praia de Boa Viagem.

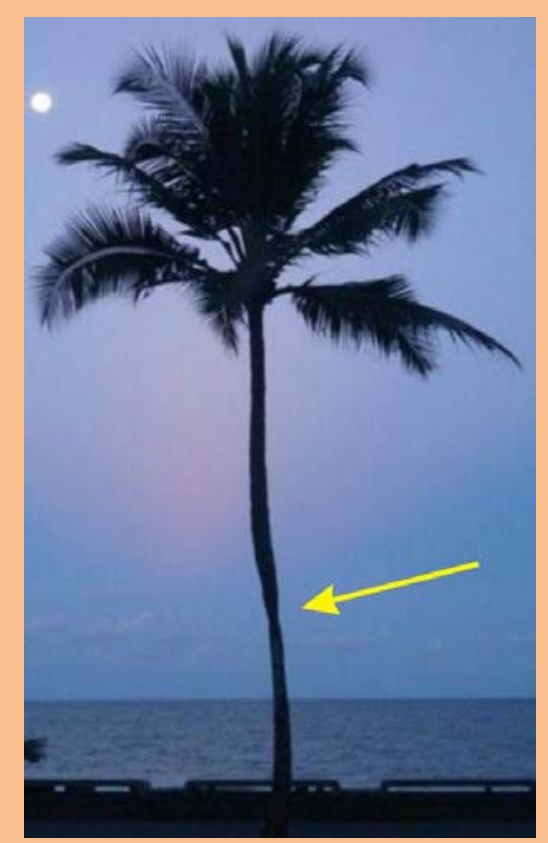

Tabela 2. Resultado da análise de correlação (r) das variáveis meteorológicas e biométricas avaliadas em áreas naturais: Praia de Maria Farinha.

\begin{tabular}{ccccc}
\hline & $\Delta \mathrm{V}(\mathrm{m} / \mathrm{s})$ & Tronco $\left(\mathrm{m}^{3}\right)$ & Folhas $(\mathrm{n})$ & $\theta\left(^{\circ}\right)$ \\
\hline$\Delta \mathrm{V}(\mathrm{m} / \mathrm{s})$ & 1 & & & \\
Tronco $\left(\mathrm{m}^{3}\right)$ & $-0,654$ & 1 & & \\
Folhas $(\mathrm{n})$ & $-0,813$ & 0,866 & 1 & 1 \\
$\theta\left(^{\circ}\right)$ & $-0,859$ & $-0,814$ & $-0,799$ & \\
\hline
\end{tabular}

Tabela 3. Resultado da análise de correlação (r) das variáveis meteorológicas e biométricas avaliadas em áreas urbanas: Praia de Boa Viagem.

\begin{tabular}{ccccc}
\hline & $\Delta \mathrm{V}(\mathrm{m} / \mathrm{s})$ & Tronco $\left(\mathrm{m}^{3}\right)$ & Folhas $(\mathrm{n})$ & $\theta\left(^{\circ}\right)$ \\
\hline$\Delta \mathrm{V}(\mathrm{m} / \mathrm{s})$ & 1 & & & \\
Tronco $\left(\mathrm{m}^{3}\right)$ & $-0,717$ & 1 & & \\
Folhas $(\mathrm{n})$ & $-0,896$ & 0,871 & 1 & 1 \\
$\theta\left(^{\circ}\right)$ & $-0,911$ & $-0,957$ & $-0,968$ & \\
\hline
\end{tabular}

Ingrid Juliane Machado de Melo et al. 
Houve uma relação significativa e inversamente proporcional entre as variáveis velocidade e direção do vento com as variáveis biométricas dos indivíduos de Cocos nucifera var. typica. Os indivíduos situados nas áreas costeiras urbanas (Praia de Boa Viagem) apresentaram menores valores de volume do tronco e do número dos folhas do que nas áreas costeiras naturais (Praia de Maria Farinha). A velocidade do vento resultante foi maior na área antrópica e houve uma modificação da direção do vento nesta mesma área em relação à área natural analisada.
Os resultados desta pesquisa inferem que os indivíduos de Cocos nucifera var. typica devem ser plantados em áreas naturais, ou em áreas com ausência de barreiras que impeçam a passagem do vento em todas as direções, como áreas próximas a construções civis ou barreiras geomorfológicas, por exemplo morros ou serras. Nestas áreas, os indivíduos terão maior número de folhas foliares, o que resulta em elevados índices de trocas gasosas (fotossíntese) e bioacumulação de nutrientes como o carbono e otimiza assim, o crescimento e desenvolvimento desses vegetais.

\section{REFERÊNCIAS}

ARTECA, R. N.; ARTECA, J. M. Characterization of gravitropic inflorescence bending in brassinosteroid biosynthesis and signaling Arabidopsis mutants. Journal of Plant Physiology, v. 168, n. 11, p. 1200-1207, 2011.

BUNCE, J. A. Performance characteristics of an area distributed free air carbon dioxide enrichment (FACE) system. Agricultural and Forest Meteorology, v. 151, n. 8, p. 1152-1157, 2011.

CASTRO, E.M.; PINTO, J.E.B.P.; MELO, H.C.; SOARES, A.M.; ALVARENGA, A.A.; LIMA JÚNIOR, E.C. Aspectos anatômicos e físiológicos de plantas de guaco submetidas a fotoperíodos. Horticultura Brasileira, v.23, n.3, p. 846-850, 2005.

CORDERO, R.A. Ecophysiology of Cecropia schreberiana saplings in two wind regimes in an elfin cloud forest: growth, gas exchange, architecture and stem biomechanics. Tree Physiology, v. 19, n. 12, p. 153-163, 1998.

DUARTE, H. M.; GEßLER, A.; SCARANO, F. R.; FRANCO, A. C.; MATTOS, E. A.; RENNENBERG, N. H.; RODRIGUES, P. J. F. P.; ZALUAR, H. L. T..; LÜTTGE, U. Ecophysiology of six selected shrub species in different plant communities at the periphery of the Atlantic Forest of SE-Brazil. Flora - Morphology, Distribution, Functional Ecology of Plants, v. 200, n. 5, p. 456-476, 2005.

DUNTEMANN, M. Elements of an Urban Tree Risk Management Program. Revista Brasileira de Arborização Urbana, v. 1, n. 1, p. 99-107, 2006.

IINO, M. Phototropism in higher plants. Elsevier Science B.V. 200. p. 659-811. 2001 
GOYAL, A.; SZARZYNSKA, B.; FANKHAUSER, C. Phototropism: at the crossroads of light-signaling pathways. Trends in Plant Science, v. 18, n. 7, p. 393-401, 2013.

MIRANDA, L. P. A.; VITÓRIA, P.; FUNCH, L. S. Leaf phenology and water potential of five arboreal species in gallery and montane forests in the Chapada Diamantina; Bahia; Brazil. Environmental and Experimental Botany, v. 70, n. 2-3, p. 143-150, 2011.

MONTEIRO, J. E. B. A.. Estimação da área foliar do algodoeiro por meio de dimensões e massa das folhas. Bragantina, v.64, n.1, p.15-24, 2005.

PELTOLA, H. M. MECHANICAL STABILITY OF TREES UNDER STATIC LOADS. AMERICAN JOURNAL OF BOTANY, V. 90, N. 10, P. 1501-1511. 2006.

RAHMAN, M. M.; KHAN, M. A. SURFACE TREATMENT OF COIR (COCOS NUCIFERA) FIBERS AND ITS INFLUENCE ON THE FIBERS' PHYSICO-MECHANICAL PROPERTIES. COMPOSITES SCIENCE AND TECHNOLOGY, V. 67, N. 11-12, P. 2369-2376, 2007.

SILVA, L. M.; MOCCELLIN, R.; WEISSHEIMER, D. I.; ZBORALSKI, A. R.; FONSECA, L.; RODIGHIERO, D. A. INVENTÁRIO E SUGESTÕES PARA ARBORIZAÇÃO EM VIA PÚBLICA DE PATO BRANCO/PR. REVISTA BRASILEIRA DE ARBORIZAÇÃO URBANA, V. 2, N. 1, P. 101-108, 2007.

SIQUEIRA, L. A.; ARAGÃO, W. M.; TUPINAMBÁ, E. A. A INTRODUÇÃO DO COQUEIRO NO BRASIL, IMPORTÂNCIA HISTÓRICA E AGRONÔMICA. EMBRAPA TABULEIROS COSTEIROS. DOCUMENTOS, 47. ARACAJÚ, 2002.

STIGTER, C. J.; MOHAMMED, A. E.; AL-AMIN, N. K. N.; ONYEWOTU, L. O. Z.; OTENG'I, S. B. B.; KAINKWA, R. M. R. Agroforestry solutions to some African wind problems. Journal of Wind Engineering and Industrial Aerodynamics, v. 90, n. 10, p. 1101-1114, 2002.

TAKAHASHI, S.; BADGER, M. R. Photoprotection in plants: a new light on photosystem II damage. Trends in Plant Science, v. 16, n. 1, p. 53-60, 2011.

VAN STAN II, J. T.; SIEGERT, C. M.; LEVIA JR., D. F.; SCHEICK,C. E. Effects of wind-driven rainfall on stemflow generation between codominant tree species with differing crown characteristics. Agricultural and Forest Meteorology, v. 151, n. 9, p. 1277-1286, 2011.

ZEIGER, E.; LINCOLN, T. Fisiologia Vegetal. 3ª Edição. Porto Alegre: Artmed, 2006. 
ZHU, J.J; LIU, Z.; LI, X.; MATSUZAKI, T.; GONDA, Y. Review: effects of wind on trees. Journal of Forestry Research, v. 15, n.2, p. 153-160. 2004.

WADT, L.H.O. Avaliação de divergência genética em coqueiro (Cocos nucifera L.) usando marcadores RAPD em amostras de plantas individuais ou compostas. 1997. 109 f. Dissertação (Mestrado em Produção Vegetal) - Centro de Ciências e Tecnologias Agropecuárias da Universidade do Norte Fluminense, Rio de Janeiro. 1997.

ZAR, J. H. Biostatistical analysis. 5th. ed. New Jersey: Prentice Hall, c2010. xiii, 944 p. 